$\overline{\overline{\text { BRUSSELS }}} \overline{\overline{\text { STUDIES }}}$

\section{Brussels Studies}

La revue scientifique électronique pour les recherches sur Bruxelles / Het elektronisch wetenschappelijk tijdschrift voor onderzoek over Brussel / The e-journal for academic research on Brussels

Notes de synthèse | 2009

\title{
Les institutions bruxelloises
}

Note de synthèse, EGB no 14

De Brusselse instellingen

The institutions of Brussels

\section{Pascal Delwit et Kris Deschouwer}

Traducteur : Micheline Goche

\section{OpenEdition \\ Journals}

Édition électronique

URL : http://journals.openedition.org/brussels/1000

DOI : 10.4000/brussels. 1000

ISSN : 2031-0293

Éditeur

Université Saint-Louis Bruxelles

Référence électronique

Pascal Delwit et Kris Deschouwer, "Les institutions bruxelloises », Brussels Studies [En ligne], Notes de synthèse, mis en ligne le 24 février 2009, consulté le 19 avril 2019. URL : http:// journals.openedition.org/brussels/1000 ; DOI : 10.4000/brussels.1000 


\section{BRUSSELS STUDIES}

www.brusselsstudies.be
ÉTATS GÉNÉRAUX DE STATEN-GENERAAL VAN CITIZENS'FORUM OF
RUXELLES RUSSEL RUSSELS

www.etatsgenerauxdebruxelles.be

Note de synthèse $\mathrm{n}^{\circ} 14$

\title{
Les institutions bruxelloises
}

\author{
P. Delwit, K. Deschouwer \\ Traduction : Micheline Goche
}

- Pascal Delwit est professeur de science politique à I'Université libre de Bruxelles (ULB). Ses travaux portent sur les partis politiques en Belgique et en Europe. Pascal DELWIT, Emilie VAN HAUTE (eds.), Le vote des Belges (Bruxelles - Wallonie, 10 juin 2007), Bruxelles, Editions de I'Université de Bruxelles, collection Science Politique, 2008.

Contact: pdelwit@ulb.ac.be, ++32 (0)2 6503903

- Kris Deschouwer est professeur ordinaire à la faculté des Sciences politiques de la Vrije Universiteit Brussel (VUB). II effectue des recherches sur la représentation des partis politiques dans des systèmes politiques complexes. Deschouwer, $\mathrm{K}$ (2009), "Coalition formation in multi-level settings : Belgium 1995 - 2008", Regional and Federal Studies, vol 19, n¹, p $13-35$

Contact: Kris.Deschouwer@vub.ac.be, ++32 (0)2 6292059

Conférence-débat :

2 mars, $19 h 45$

Flagey

Place Sainte Croix 1050 Bruxelles 


\section{Constats}

Le statut actuel de Bruxelles n'est pas le résultat d'un choix délibéré. II ressemble en cela à celui de la Belgique en général : personne ne l'a voulu tel qu'il est, personne ne trouve qu'il représente une solution souhaitable.

Les institutions belges et bruxelloises ont été créées sur la base de la frontière linguistique fixée en 1963 (la question restant posée de l'éventualité de modifier cette frontière), d'un règlement territorial de l'emploi des langues (assorti d'exceptions le long de la frontière et autour de Bruxelles) et de la séparation systématique des deux grandes communautés linguistiques. Bruxelles est un élément essentiel du compromis à la belge relatif à la réorganisation du territoire.

\section{Régions et Communautés}

Ce rôle de Bruxelles dans le compromis à la belge s'exprime en premier lieu par la présence, d'une part, d'institutions relatives à des matières régionales et, d'autre part, d'institutions qui se rapportent aux communautés. La double fédération de régions et de communautés que Bruxelles est devenue ne constitue pas non plus la concrétisation d'un projet, mais plutôt une réponse à l'existence de deux conceptions différentes de l'organisation territoriale du pays. En fait, ce sont la localisation de Bruxelles et l'évolution de l'emploi des langues à Bruxelles qui ont conduit à la création, en 1970, des régions et des communautés. La préférence affichée du côté flamand pour les communautés se fondait sur l'hypothèse selon laquelle Bruxelles ferait également partie de la Communauté flamande, tandis que la prédilection manifestée du côté francophone pour les régions était inspirée par le souhait de voir Bruxelles comme une entité indépendante, une région à part entière. La double fédération belge a donc engendré, à Bruxelles, les institutions de la Région, la VGC, la COCOF et la GGC/COCON. 
Le problème de Bruxelles a toujours influencé fortement les débats institutionnels ; citons, en particulier, l'échec du Pacte d'Egmont et de l'Accord du Stuyvenberg en 1977-1978 ainsi que la décision prise en 1980 de mettre Bruxelles « au frigo » jusqu'au compromis de 1988. Les débats portaient invariablement sur la limite de Bruxelles et sur la signification de celle-ci, sur le statut régional et sur la place et le rôle de la minorité néerlandophone dans la région. Finalement, une structure complexe a été créée, la Région de Bruxelles-Capitale, dont le droit à l'existence en tant que région à part entière est toujours mis en question par la plupart des responsables politiques flamands et dont les limites sont toujours contestées par la majorité des responsables politiques francophones.

La différence de conception des deux groupes linguistiques quant au rôle de Bruxelles dans les institutions belges se retrouve aussi dans l'asymétrie qui existe entre la COCOF, institution législative indépendante, et la VGC, élément de la Communauté flamande (en Belgique).

\section{La région et les communes}

Sur la toile de fond des tensions relatives à la langue et à son emploi, à Bruxelles, certains débats institutionnels ont été prudemment évités, voire carrément non engagés, en particulier au niveau communal. La grande opération de fusion de 1976 est passée à côté de Bruxelles parce qu'à ce moment-là aucune solution acceptable ne pouvait être trouvée. Le débat sur les fusions en Belgique portait sur la capacité locale de gestion, sur l'efficacité et sur des économies d'échelle. À Bruxelles, le statut de la région elle-même n'était pas encore clairement défini, ce qui rendait impossible toute discussion constructive sur le rôle qu'y joueraient les communes.

Du côté flamand, on parlait de réduire le nombre de celles-ci, voire de les grouper toutes en une seule. Cette idée partait du constat de la faible représentation des néerlandophones dans de nombreuses communes, minorité qui ne pourrait être efficacement protégée qu'au sein d'un ensemble plus large. L'inspiration communautaire des propositions relatives au niveau communal empêchait, à l'époque, tout débat rationnel à leur sujet.

Un tel débat s'impose toutefois à propos de la configuration des communes bruxelloises et de la répartition des compétences entre celles-ci et la région, débat justifié notamment par les conceptions nouvelles de la politique de la ville et par la réalité du paysage communal de Bruxelles. En effet, celui-ci s'est fortement diversifié sur trois plans au moins : la richesse, l'étendue et la démographie (voir tableau 1).

En ce qui concerne la richesse, les différences sont énormes. Commune la plus pauvre du pays, Saint-Josse atteint à peine la moitié de l'indice de bien-être moyen des communes belges. Mais sept communes bruxelloises dépassent la moyenne de 100. II va de soi que cette configuration a un impact sur les revenus et donc aussi sur les dépenses des communes.

En ce qui concerne l'étendue, la diversité est tout aussi importante. La commune la plus grande (Bruxelles) couvre une superficie égale à 29,6 fois celle de la commune la plus petite (Saint-Josse). On compte aussi six communes d'étendue inférieure à 5 $\mathrm{km}^{2}$ et quatre qui dépassent $15 \mathrm{~km}^{2}$. 
Quant au chiffre de la population, il présente également des différences impressionnantes. La commune la plus étendue (Bruxelles) compte 7,83 fois plus d'habitants que la commune la moins peuplée (Koekelberg). Et la densité de population de Saint-Josse est 10,8 fois plus élevée que celle de Watermael-Boitsfort. Notons que ces chiffres ne concernent que les habitants effectivement inscrits au registre de la population.

Devant de telles divergences observées sur un territoire urbain exigu, une question s'impose : peut-on encore administrer le niveau communal de la façon dont on gère habituellement une commune en Belgique?

\section{La représentation politique}

L'histoire de la Belgique a influencé et influence encore fortement la détermination des institutions tant régionales que communales. A l'origine de la Région de Bruxelles-Capitale, il y a plusieurs visions du problème linguistique et celles-ci se manifestent dans ses institutions et ses limites. C'est pourquoi ces institutions complexes et hybrides sont fondées, en grande partie, sur la différence entre les deux groupes linguistiques. Ceux-ci sont dûment séparés l'un de l'autre - notamment pour l'organisation des élections - et sont ensuite obligatoirement réunis pour cogérer la ville. En ce sens, Bruxelles ressemble fort à la Belgique, où les mêmes principes de nette séparation et de collaboration obligée sont appliqués. 


\section{Questions-problèmes}

Les principaux problèmes que posent les institutions bruxelloises sont liés à leur inadéquation à la réalité urbaine et régionale du 21è siècle. L'influence de l'histoire a conduit, en effet, à créer des institutions qui répondent aux données et aux problèmes datant de quelques décennies. Cependant, aujourd'hui, on ne peut faire abstraction ni de l'importance du problème des langues et de leur emploi ni des relations asymétriques entre les langues.

\section{La représentation politique}

La Région de Bruxelles-Capitale est définie comme une région composée de deux groupes nettement distincts l'un de l'autre parce qu'ils ne parlent pas la même langue. Ce point de départ pose au moins deux types de problèmes. Le premier concerne la frontière stricte qui sépare les deux groupes linguistiques, dont l'existence laisse supposer que chaque Belge peut, voire devrait, se considérer comme faisant partie soit de l'un, soit de l'autre. L'absence de choix ne peut être traduite institutionnellement. Les élections pour le Parlement de la Région de Bruxelles-Capitale illustrent particulièrement bien ce phénomène. En effet, un mouvement politique qui voudrait s'adresser à tous les habitants de la région ne le pourrait pas.

L'expérience des élections pour le Conseil d'Agglomération de 1971 - où les garanties d'une présence néerlandophone suffisante n'étaient pas solides et pouvaient donc être facilement contournées - a entraîné la suppression des listes bilingues pour l'élection des représentants bruxellois et l'obligation pour les candidats de faire, une fois pour toutes, un choix linguistique. Par conséquent, aujourd'hui, les partis s'adressent en général à leur propre groupe linguistique, bien que la plupart des partis néerlandophones aient également des contacts avec les électeurs francophones, et même si, pour une partie de la population, l'appartenance à une seule des communautés est ambiguë ou peu importante.

Ce modèle, qui diffère donc de celui qui régit l'organisation des élections communales, a conduit à un compromis remarquable pour la composition du Parlement bruxellois. Au nombre de 89, les représentants sont incontestablement trop nombreux en termes absolus, mais ce fait résulte, d'une part, du désir d'assurer une représentation parlementaire décente aux Néerlandophones et, d'autre part, de la volonté de maintenir malgré tout un lien entre la taille des communautés et leur poids parlementaire.

Cette stricte répartition de la population en deux groupes linguistiques pose un deuxième problème. En effet, elle n'a véritablement de sens que pour les habitants d'origine belge. Or, au cours de ces dernières décennies, la composition de la population bruxelloise s'est progressivement diversifiée et internationalisée. Pour un nombre croissant d'habitants de Bruxelles, les institutions fondées sur (l'opposition entre) les groupes linguistiques belges ne représentent pas grand-chose. Le fait est d'importance car il constitue une zone d'ombre dans les débats sur Bruxelles. À cause de cette composition très spécifique de la population de la Région de Bruxelles-Capitale, des groupes très importants d'habitants ne jouissent pas du droit de vote. Ne perdons pas de vue que plus de $26 \%$ de la population bruxelloise n'a pas la nationalité belge. Cela atténue peut-être l'inadéquation entre les institutions et la 
composition de la population, mais cela pose pour Bruxelles un gros problème de légitimité démocratique.

\section{Région et communauté}

Dans le contexte belge, les compétences propres aux communautés sont liées à la langue. Elles ne font donc pas partie de l'ensemble des compétences de la Région de Bruxelles- Capitale. On peut cependant se demander si, pour assurer une gestion efficace et cohérente de la région, il ne faudrait pas attribuer aussi à Bruxelles des compétences communautaires. La région peut-elle prétendre figurer sur la carte internationale des villes sans être compétente en matière de tourisme ? Peut-elle assumer avec toute l'autorité voulue ses compétences relatives à l'économie et au marché du travail - appelées à devenir plus importantes encore dans l'avenir - sans se voir attribuer en même temps une compétence en matière d'enseignement ? On peut aussi se demander s'il n'est pas envisageable que la Région de Bruxelles-Capitale mène une politique dans les domaines de la culture, du bien-être, des soins de santé, de l'intégration sociale,... Les tabous et l'angoisse de la minoration sont très importants à ce propos.

\section{La région et les communes}

L'organisation et le fonctionnement des communes de la Région de Bruxelles-Capitale reflètent encore les choix opérés pour toutes les communes belges il y a longtemps, bien avant qu'il soit question de décentralisation en régions et communautés. Seules des modifications marginales y ont été apportées, notamment en matière de garanties pour les néerlandophones. La remarquable créativité dont les élites politiques belges et bruxelloises ont fait preuve pour trouver des solutions aux tensions de nature communautaire présente un contraste frappant avec l'absence d'imagination et d'audace observée lors de l'organisation du niveau communal. Cette pusillanimité pourrait bien être due au manque d'indépendance du niveau régional par rapport au niveau communal. La plupart des membres du Parlement de la Région de Bruxelles-Capitale exercent également un mandat au niveau communal, en tant que membre du conseil communal, échevin ou bourgmestre. Ils font ainsi partie de leur propre autorité de tutelle et de l'autorité chargée éventuellement de redéfinir leur mandat ou la portée de celui-ci. 


\section{Pistes politiques}

Les options possibles de modification et d'amélioration des institutions découlent logiquement des constats et problèmes mentionnés plus haut. On peut en déduire tout d'abord que la simplification des institutions n'est pas une tâche facile. La complexité a une raison et une histoire et l'adaptation des institutions bruxelloises à la ville actuelle pourrait augmenter encore cette complexité. Toutefois, il faut aussi pouvoir réfléchir sur la ville sans tabous.

\section{L'organisation des élections}

Comme nous l'avons vu, le système électoral qui s'applique à la Région de Bruxelles-Capitale est le reflet du passé et de la crainte des néerlandophones de ne pas être suffisamment représentés dans les organes de gestion. Ce système présente, par conséquent, un déficit démocratique auquel il n'est pas simple de remédier, surtout lorsqu'il s'agit précisément de garantir la présence néerlandophone.

En principe, il est facile de résoudre le problème de la non-représentation d'un groupe important d'habitants privés du droit vote. II suffit - bien que cela demande une modification de la législation fédérale - que tous les habitants de la région qui jouissent du droit de vote pour les élections communales se voient accorder également ce droit pour l'élection au Parlement de Bruxelles-Capitale. Ainsi s'accroît en même temps le groupe des personnes pour lesquelles le choix difficile de l'un des deux groupes linguistiques n'est pas nécessaire.

La tâche n'est pas aisée. Voici quelques possibilités. Le changement le plus radical consiste à supprimer la répartition en groupes linguistiques pour les élections, tous les électeurs ayant alors accès à toutes les listes. Mais alors la protection des néerlandophones disparaît. Les élections pour le parlement régional ressemblent alors aux élections communales, où les néerlandophones peuvent être élus sur des listes linguistiquement mixtes, mais avec une représentation beaucoup plus faible. Cependant, on peut aussi opter soit pour l'établissement de quotas fixes pour les deux groupes linguistiques, soit pour la détermination d'un quota minimum pour les néerlandophones. Une variante de cette procédure consiste à attribuer un certain nombre de sièges fixes à un groupe " ouvert » ou « non défini ", à côté du quota fixe accordé aux deux groupes linguistiques. Mais ceci perturbe à nouveau les équilibres établis dans la composition paritaire du gouvernement de la Région de BruxellesCapitale.

Une autre option, plus " douce ", serait de maintenir le caractère unilingue des listes mais de donner deux voix à chaque électeur, ce qui permettrait à celui-ci de franchir la frontière qui sépare les groupes linguistiques. Cette option aurait l'avantage d'entraîner les hommes politiques à prendre également en compte les idées défendues par le groupe linguistique qui n'est pas le leur, mais elle présenterait l'inconvénient de perpétuer la nette séparation des deux groupes. De plus - étant donné la forte suprématie numérique des francophones - les relations entre les partis néerlandophones seraient beaucoup plus fortement influencées par les électeurs francophones que l'inverse. Malheureusement, en cette matière, il n'existe pas de solution prête à l'emploi qui ne suscite immédiatement d'autres questions et préoccupations. 


\section{Région et Communautés}

Il faudrait examiner la possibilité d'attribuer également à la Région de Bruxelles-Capitale des compétences communautaires, bien que la chose soit tout aussi malaisée et exige une révision de la Constitution fédérale. Cette attribution reviendrait à créer une communauté supplémentaire plutôt qu'à supprimer la Communauté flamande et la Communauté française. Du côté de la Communauté flamande, se posera toujours la question pertinente du traitement des matières personnalisables en néerlandais pour les Bruxellois qui le souhaitent (enseignement, soins de santé, arts). Les services prestés par la communauté bruxelloise - et l'enseignement en particulier peuvent et doivent évidemment être multilingues. C'est-à-dire : au moins bilingues, mais de préférence ouverts aux autres langues qui sont parlées aujourd'hui à Bruxelles.

\section{Région et communes}

Il est urgent pour la Région de Bruxelles-Capitale de voir s'instaurer une réflexion approfondie sur le rôle et la signification des petites entités d'une région qui est, en fait, une ville et qui peut donc donner un autre contenu au concept de « commune " que la Wallonie et la Flandre. Ce débat doit être mené en profondeur, mais aussi avec la prudence nécessaire. N'oublions pas que la commune la moins peuplée de la Région de Bruxelles-Capitale est plus grande que la commune moyenne de Flandre ou de Wallonie. Avec un nombre d'habitants égal à celui de la région de Bruxelles-Capitale, le Brabant flamand est divisé en 65 communes. Et dans le Brabant wallon - dont la population est le tiers de celle de Bruxelles - il y a 27 communes. Ces chiffres relativisent la " nécessité » souvent défendue, surtout du côté flamand, de réduire le nombre des communes bruxelloises.

II ne faut pas perdre de vue non plus que les fusions effectuées, dans le passé, dans un environnement urbain ont généré des problèmes. La fusion opérée à Anvers a entraîné la création de districts. On connaît les effets pervers occasionnés par la fusion effectuée à Charleroi. II est important que les services publics soient proches du citoyen et que les électeurs soient proches de leurs mandataires élus. Et on ne peut ignorer le fait qu'une partie non négligeable de la population - tout comme en Flandre et en Wallonie - se sent liée à la commune dans laquelle elle habite.

Pour conclure, répétons que le débat restera difficile à cause de sa dimension communautaire, même si l'Accord du Lombard a œuvré, grâce à un incitant financier, à assurer beaucoup mieux la présence flamande dans les organes de la gestion de la commune.

Mais Bruxelles a acquis entre-temps, tout comme les autres communes, la compétence de définir et d'organiser elle-même le niveau communal. Aujourd'hui, à ce propos, se posent des questions relatives non seulement aux moyens financiers mais aussi aux rapports entre la Région actuelle et les communes. La question de la façon dont les deux niveaux doivent ou peuvent être gérés reste ouverte.

II existe divers exemples de régions urbaines - notamment Berlin et Vienne - où les rapports entre ville, région et entités plus restreintes sont réglés de manière réfléchie. La région doit pouvoir parler d'une seule voix vers l'extérieur, par exemple lors d'entretiens avec les autres régions belges. En son propre sein, il peut y avoir place pour des divergences en matière de politique et de prestation de services. 
Dans ce contexte, trois questions liées entre elles doivent recevoir une réponse concrète. La première concerne la signification de la commune elle-même, sa mission et ses compétences, et aussi la mission et les compétences les plus susceptibles d'être assurées, de la même manière, par les autorités régionales, dans l'ensemble de la région. Pour cela, il faut garder à l'esprit les avantages et les inconvénients du principe de subsidiarité et ne pas supposer naïvement qu'il n'y a d'opposition communautaire sur ces sujets ni à l'intérieur ni à l'extérieur de Bruxelles.

La deuxième question concerne le nombre et les limites des communes. Leur nombre et limites actuels sont ceux des villages devenus une ville, mais ils ne suivent pas les lignes que la mise en forme de celle-ci a tracées entre-temps, à l'exception de l'élargissement de Bruxelles-ville. Nous avons montré que d'un point de vue socio-économique, socio-géographique et socio-politique, cette réflexion est plus que judicieuse.

Troisièmement, se pose la question de la façon dont les communes peuvent être gérées. Si l'on réfléchit sans tabous et sans se braquer sur les vieilles institutions et procédures familières, si l'on veut donc un nouveau type de commune pour la villerégion, on peut certainement investiguer des moyens plus créatifs d'impliquer, de façon plus intensive et plus permanente, le citoyen dans la politique directement liée à l'organisation de la ville et à la prestation de services là où les Bruxellois résident et vivent.

\section{Références}

DELWIT, P., REA, A., SWYNGEDOUW, M. (eds.), Bruxelles ville ouverte, Paris, L'Harmattan, collection Villes et entreprises, 2007.

WITTE, E., ALEN, A., DUMONT, H., VANDERNOOT, P. a DE GROOF, R. (eds) (2003), De Brusselse negentien gemeenten en het Brussels model / Les dix-neuf communes bruxelloises et le modèle bruxellois, Brussel / Bruxelles: Larcier

WITTE, E., ALEN, A., DUMONT, H. \& ERGEC, R. (eds) (1999), Het statuut van Brussel / Bruxelles et son statut, Bruxelles / Brussel: Larcier 


\section{Annexes}

\begin{tabular}{|c|c|c|c|c|}
\hline & $\begin{array}{l}\text { Superficie en } \\
\text { km2 }\end{array}$ & Population & $\begin{array}{l}\text { Densité de } \\
\text { population }\end{array}$ & $\begin{array}{l}\text { Indice de } \\
\text { bien-être }\end{array}$ \\
\hline Anderlecht & 17,7 & 99.085 & $5.286,70$ & 77 \\
\hline Auderghem & 9 & 30.086 & $3.239,60$ & 108 \\
\hline Berchem Sainte-Agathe & 2,9 & 20.976 & $6.769,80$ & 102 \\
\hline Bruxelles & 32,6 & 148.873 & $4.381,20$ & 76 \\
\hline Etterbeek & 3,1 & 49.902 & $13.049,50$ & 82 \\
\hline Evere & 5 & 34.727 & $6.589,90$ & 88 \\
\hline Forest & 6,2 & 48.906 & $7.611,10$ & 86 \\
\hline Ganshoren & 2,5 & 21.743 & $8.394,00$ & 101 \\
\hline Ixelles & 6,3 & 79.768 & $12.251,20$ & 87 \\
\hline Jette & 5 & 44.601 & $8.377,40$ & 94 \\
\hline Koekelberg & 1,2 & 19.020 & $15.114,20$ & 83 \\
\hline Molenbeek Saint-Jean & 5,9 & 83.674 & $13.327,00$ & 65 \\
\hline Saint-Gilles & 2,5 & 45.235 & $17.321,80$ & 67 \\
\hline Saint-Josse-ten-Node & 1,1 & 24.078 & $20.259,20$ & 52 \\
\hline Schaarbeek & 8,1 & 116.039 & $13.559,60$ & 70 \\
\hline Uccle & 22,9 & 76.732 & $3.272,60$ & 110 \\
\hline Watermael-Boitsfort & 12,9 & 24.134 & $1.880,00$ & 114 \\
\hline Woluwé Saint-Lambert & 7,2 & 49.261 & $6.622,30$ & 102 \\
\hline Woluwé Saint-Pierre & 8,9 & 38.651 & $4.284,10$ & 112 \\
\hline
\end{tabular}

Tableau 1: Superficie, population et bien-être des communes bruxelloises (2005) Source : Institut national de Statistique 